\title{
Some paradigms for coordinating feedback control with applications to urban traffic control and smart grids
}

\author{
René Boel, Nicolae Marinica, Mohammad Moradzadeh, Herman Sutarto ${ }^{1}$ \\ SYSTeMS Research Group, \\ Ghent University \\ Technologiepark-Zwijnaarde 914, B9830 Gent, Belgium \\ Rene.boel@ugent.be
}

\begin{abstract}
This lecture treats the problem of designing local control agents for cooperating components in a network of interacting dynamic systems. Each local control agent must ensure that all local specifications are met, but at the same time must ensure that the different components help each other in achieving good global behavior as well as good local behavior. This problem will be illustrated by using urban traffic control and smart electric power grids as examples. Centralized or hierarchical control approaches are not robust against failures in communication networks, and require unrealistic assumptions on the knowledge of each agent about the overall model. A completely decentralized approach, where each local control agent selfishly tries to achieve its local specifications only, runs a high risk of global interactions that may destabilize the system, making it impossible to achieve the specifications. This talk proposes two paradigms for distributed feedback control that require very little information exchange and very little global model knowledge. The leader/follower control paradigm is illustrated for urban traffic control: heavily loaded leader agents send messages to their follower neighbors requesting that these followers give green only to platoons of vehicles travelling towards the leader intersection at those times when this will be optimal for the performance of the leader. Another coordination paradigm is called the coordinating model predictive control (CMPC). Consider a power transmission network that has been partitioned in interacting regions, where CMPC is used in order to prevent the spread of the disturbances following incidents like line or machine failure. CMPC tries to resolve this by having each local control agent apply a model predictive controller, using as on-line available information not only the local voltage and current measurements, but also information on the planned sequence of future control actions of neighboring agents, communicated to it from time to time. This talk will discuss some of the minimal requirements for modeling, communication and control agent set-up in order to robustly achieve specifications using distributed control.
\end{abstract}

Keywords; distributed control; networked systems; urban traffic control; power systems control; leader/follower; model predictive control

1) Herman Sutarto is currently at Dept. of Electrical Engineering, Institut Teknologi Harapan Bangsa, Bandung 40132, Indonesia

\section{INTRODUCTION}

\section{A. Problem motivation}

This lecture treats the problem of designing local control agents for cooperating components in a network of interacting dynamic systems. Each local control agent must ensure that all local specifications are met, but at the same time must ensure that the different components help each other in achieving good global behavior. This problem will be illustrated using urban traffic control and smart electric power grids as examples, but many other large systems applications can be considered including irrigation/flood control, freeway traffic, logistics, autonomous vehicles sharing tasks.

Traditional control design starts from a given plant, with its known dynamical model. If analysis shows that this plant does not achieve the desired specifications then the control engineer designs a feedback controller. This feedback controller is nowadays implemented as an algorithm, that is executed concurrently with the plant evolution, by a control agent (typically a computer processor). From time to time the control agent (CA) receives information from sensors approximately measuring the value of variables characterizing the plant. The CA uses this information, together with its knowledge about the plant model, in order to decide what control values must be applied at the actuator in order to satisfy all the specifications imposed on the closed loop plant behavior. It is known that the feedback nature of the control loop makes the performance of the controlled system robust against modeling errors, sensor inaccuracies, and many other faults.

Many plants nowadays are so large that centralized control by one single CA is not realistic (either the off-line controller design problem or the on-line control agents calculation or both may be computationally too expensive) nor is it robust against communication errors. Examples are traffic networks (urban as well freeway), irrigation channels (or 
dually flood control), communication networks, logistics networks, and electrical power nets (sometimes called the largest man-made system). These large plants are often modeled as networks of interacting local components, each component being controlled by a separately designed local control agent. This talk discusses some methods for designing local CAs that cooperate with each other so as to guarantee globally good performance of the overall system.

The following limitations of typical applications make this problem challenging. Each component has local sensors that provide data that the local CA can use for local feedback actions (e.g. at each intersection in an urban traffic network a local CA uses detectors of oncoming traffic to select the switching times of the traffic lights), but these data sets are often so large that it is impractical to distribute them throughout the whole network. Moreover CAs can communicate only with their neighbors via an unreliable communication network with limited bandwidth, and the closed loop control system should be robust against failure of the communication channels. Each local control agent knows a dynamic model of its own component, but often has limited information only on the overall network model (e.g. it only knows a coarse model of its neighbors and of how it interacts with its neighbors). This is due to the impossibility in a large network to maintain a coherent view of all the millions of small changes that are made to the system (in an urban traffic network capacity reductions due to repair works or due to incidents are often not known globally; in power systems the post-mortem analysis of blackouts often shows that the reason why the system failed was the fact that one region did not know that a facility in a neighboring region was out of operation at the time of an incident). Hence measurements and plant knowledge are often local only, while the specifications to be met by the closed loop system typically are global, e.g. achieve low average delay for all vehicles, with priority for high priority vehicles, or maintain the voltage of each bus in the global power system within its specified bounds.

Centralized or hierarchical approaches require reliable message exchange over communications networks with high bandwidth. A completely decentralized approach on the other hand implies that the global specification must be translated by a supervisor into local specifications sent to local control agents via a limited bandwidth communication network. It is obvious that if each control agent selfishly tries to achieve its local specifications only without taking into account what effects these actions have on the neighbors then there is a high risk that the interactions between components (dynamical model of each component with its local controller) may destabilize some loops in the network, causing failure to achieve the system specifications.

This paper discusses two distributed control paradigms that improve system performance by coordinating the actions of local control agents while requiring very little communication between neighboring control agents, minimal intervention of the supervisor, and limited global model knowledge at each control agent. Messages that a control

This research was supported by the European Union FP7 programme under contracts $\mathrm{C} 4 \mathrm{C}$ and DISC, and by the Research Council of Ghent University agent $\mathrm{CA}_{\mathrm{i}}$ sends to neighboring control agents carry information on planned control actions by agent $\mathrm{CA}_{\mathrm{i}}$, or information that updates the local cost function and specifications of a neighboring control agent, so as to achieve cooperative behavior among the CAs.

\section{B. Leader/follower paradigm}

The first coordination approach is called leader/follower control. It is most easily explained by using as an example urban traffic control for networks where the load is initially very light, but starts building up at the start of the morning rush hour to an intermediate load. Then the load eventually reaches a level where at a few heavily loaded intersections long queues will build up that block upstream intersections. This may lead to global deterioration of the performance, even to gridlock. Thus it is natural that for these intermediate load conditions the supervisor selects the most heavily loaded intersections, and assigns their CAs the role of leaders, that send messages to the neighboring follower CAs. All intersections that are not leaders are controlled by follower CAs. In order to avoid global performance deterioration the leader agents must at all cost avoid wasting capacity, i.e. leader agents must avoid giving green to directions of traffic flow where there are no arriving vehicles. This waste of capacity can be reduced by releasing platoons of vehicles at neighboring follower intersections only at such times that (almost all) the platoons arrive at the leader intersection during a green period. In this way the leaders try to synchronize the signal switching times of all their upstream followers so that two opposite directions of two-way traffic flow have platoons arriving during the same green interval. This avoids waste of capacity e.g. because a traffic has to give green to a large platoon of vehicles travelling North-South at a time when there is no South-North traffic using the green. In order to achieve this coordination each leader CA sends messages to its neighboring follower CAs specifying the intervals of time in the near future when platoons of vehicles should be given green at the follower (taking into account the known model information of the delay for vehicles travelling from follower to leader), and if this information is used as an additional constraint in the local optimization used by the followers in order to make their local feedback control decisions, then this can significantly reduce the risk of gridlock. This has been shown via simulation for simple networks.

In general the leader/follower approach can be used for coordinating actions of local feedback controllers that approximately minimize a local cost function subject to constraints (e.g. a model predictive controllers). Leaders then send messages to followers containing instructions on how to adjust the local cost function at the follower so as to ensure that the actions of the followers make it easier for the leaders to achieve a low cost. This coordination between leaders and followers makes it easier for the overall control system to achieve its specifications as long as the additional specifications sent to the followers do not cause overload at the followers. This may happen if a follower has more than one leader, and these leaders send contradictory specifications. Thus the leader/follower approach is suited well for 
intermediate load cases, not for heavily loaded systems. The leader/follower approach can handle easily the randomness in the evolution of intermediate load systems. For heavily loaded systems the noise is often negligible in comparison to the average load, thanks to the law of large numbers. Then other coordinating strategies must be used for networked control design.

\section{Coordinated Model Predictive Control (CMPC)}

Another obvious limitation of the leader/follower paradigm is that components should know at each time which side of a link connecting 2 neighboring components determines the value of the variable linking these 2 components. In the urban traffic case an intersection knows that traffic flows from its upstream intersections towards itself, and it knows also which flows of vehicles it sends to downstream intersections. In an electrical power transmission system this assumption is not valid: active, resp. reactive power, can flow in either direction along a transmission line (also called tie-line). Depending on the load (and the local voltage) of each component either one of the regions connected by the tie-line can send active, resp. reactive, power to the other component in order to help this neighbor to maintain its frequency, resp. its voltage, within specified limits. The leader/follower assignment should be updated so frequently that all the effort for the feedback control is delegated to the supervisor, beating the purpose of the distributed controller. The communication between local sensors and supervisor would then become the bottleneck. Therefore another coordination paradigm, coordinating model predictive control (CMPC) is proposed below, and explained using long term voltage stabilization in a power transmission network as an example.

Assume that each region in the power system is equipped with an anticipating feedback controller, like a model predictive controller. This means that the local control agent uses local observations to estimate the local state; then the local CA uses this state estimate in order to calculate (e.g. via simulation over a given time horizon starting in the estimated current state) what the effect will be of different possible future control actions; then the CA compares these different possible control sequences and applies the first control value of the best choice to the actuator of local component. This is possible only if the control agent knows what will be the future flow of active, resp. reactive, power to be expected over each tie-line that connects it to a neighboring region. If this interaction with neighboring regions would not be known then it is likely that neighboring regions counteract each other's feedback control actions. For example if the voltage drops on both sides of a tie-line as a result of a shortage of reactive power in one of the two regions then both regions may increase their output of reactive power proportionally, thereby overreacting (generating together more than the required reactive power) eventually causing an increase in voltage, which will in turn be compensated by both regions reducing their generation of reactive power, and so on. It is clear that this can lead to destabilizing oscillations. Such destabilizing feedback loops may occur even in cases where a simple centralized controller would easily solve the problem. The reader should compare this cause of instability to the classical explanation of the Nyquist criterion: in the case of a single feedback loop time delays of the interactions may cause an $180^{\circ}$ phase shift, leading to destabilizing positive feedback. In the case of interacting components forming a loop the time delay may be due to delays in the interaction between neighboring components, but the effect of positive feedback over a loop is the same.

This destabilization can be avoided if neighboring control agents inform each other of their planned future control actions, and if each control agent knows enough about the model of its neighbors to understand what the approximate effect of these control actions will be for its own tie-line variables. If the prediction horizon of each local MPC is long enough to predict loop effects (how a neighbor is going to react to its own planned control actions) then this exchange of information can avoid destabilizing control loops in the controlled plant. This CMPC approach will be illustrated below for voltage control in the electric power transmission grid.

\section{Comparison of coordination paradigms}

Clearly this CMPC paradigm is more flexible than the leader/follower paradigm in terms of allowable interactions among components, but it does require more detailed knowledge about the neighbors' model, and it is usually computationally more expensive. Which coordination paradigm is most suitable depends on the application. And besides these two paradigms one can imagine many other ways of organizing the communication exchange between local control agents so that the actions of these neighboring control agents are coordinated, thus avoiding destabilizing loops. We believe that development of novel coordination paradigms, and better insight in when to use a particular coordination paradigm, can only come a systematic study of more application examples.

It should also be observed that proving stability is in general difficult due to the large number of loops in a typical network. Passivity based approaches show most promise for such a stability analysis.

\section{URBAN TRAFFIC CONTROL USING THE LEADER/FOLLOWER PARADIGM}

\section{A. Role of leaders and followers in urban traffic control}

Open loop control of traffic lights in an urban environment is typically tuned so as to generate green waves, where the switching times at successive traffic lights are shifted by the time delay of vehicles traveling along the most critical axes of traffic flow so that vehicles cross consecutive intersections without ever having to stop, in the ideal case without noise. Local feedback control tries to compensate for the random perturbations, and to further reduce the local queueing time, at each intersection by adjusting the switching times of the traffic lights to the actually observed local arrival times of vehicles. This tends to destroy this green wave synchronization. 
Combining a green wave with feedback control thus requires active coordination among the neighboring control agents.

This problem is not critical when the traffic load is very light, since then all specifications are very easy to meet. It is also not relevant under very heavy load since then the effect of the random perturbations is negligible compared to the average load. However under intermediate load, when there are only a few critical intersections that have difficulty achieving their local specifications, this problem becomes relevant. It is then a good solution to designate these critical intersections as leader intersections, and to allow these leader intersections to generate additional specifications on the time intervals during which they allow platoons of vehicles to start traveling from the neighboring follower intersections towards this leader.

The roles of leaders and followers are assigned by a higher level coordinator, and in this paper we assume the leader/follower assignment is known. The goal of the coordination strategies is to make it easy for the leaders to satisfy their goals - whether these goals are formulated via local specifications or based on the on-line optimization of local cost criteria. Since the overall cost of the system depends critically on the performance of the leaders this coordination paradigm will ensure also good overall system performance, as long as the leader/follower assignment corresponds to the current overall network since by definition followers can always satisfy their goals easily.

The goal is in other words the design of a control agent for each intersection such that local congestion is limited and does not spread to neighboring intersections causing gridlock. Intuitively the uncontrolled spread of congestion in the overall network can be avoided by a feedback coordinator that allows the leader intersections to minimize their waste of capacity that results when green light is given to a direction where there is currently (in some of the approach directions) no queue nor any arriving platoon. This good behavior at the leader may possibly be at the expense of some waste of capacity at the followers, since followers are so lightly loaded that even with an inefficient control strategy they do not develop long queues. This coordination strategy will reduce the risk that the network becomes overloaded, as long as the ensuing waste of capacity at the follower components is not so large as to make these followers overloaded.

\section{B. How to design a good control agent}

The coordinating feedback CAs in this problem select the red/green switching times of traffic lights (not just the cycle time, or the red/green split) as actuating signal, for a case study consisting of a Manhattan grid of intersections that are connected by 2 -way road links. Each local controller needs sensors detecting approximately the arrival times of vehicles. Thus a model that describes traffic at the granularity of a few seconds is needed. Microscopic models that describe the movement of each vehicle however lead to models that are computationally too expensive for prediction of the future behavior over a sufficiently long time horizon. The model that is most appropriate for this analysis describes traffic using platoons of vehicles traveling close together at approximately the same speed.

Assume that at time $t$ there are $K(t)$ platoons, Platoon $_{k}, k=$ $1, \ldots, K(t)$, present in the network under study. The state $X_{t}$ of the system at time $t$ is obtained by specifying for each of these $K(t)$ platoons its location $\operatorname{Loc}_{k}(t)$ of the first vehicle of Platoon $_{k}$ and the number Size $_{k}(t)$ of vehicles in this $k$-th platoon. While traveling along a road link connecting 2 intersections the platoons incur a random delay depending on the allowable speeds, consistent with the local traffic density. Randomness is furthermore introduced in the evolution of the system because at intersections platoons split up randomly according to the destination of the vehicles, because the platoon size and speed can change along a road link (e.g. because of parked vehicles, or side roads without sensors), and due to the random arrival times and random sizes of the platoons entering the network under study at its edges.

The evolution of the traffic state also depends on the switching times of the traffic lights, which is the control action under consideration. The goal of the feedback controller implemented by the CA at a critical intersection, a leader agent, is to minimize the waste of capacity at this intersection, averaged over all flow directions for that intersection. Knowledge of (an estimate of) the state of the platoon based model allows prediction of the evolution over a given time horizon of the queue sizes at each intersection, for each given sequence of switching times of the traffic lights (analytically in simple cases, or via simulation). Based on these simulated trajectories it is possible to verify whether the behavior corresponding to a given sequence of switching times of the traffic lights, over a given prediction horizon, satisfies all specifications. It is also possible to evaluate the average waiting time, and many other cost functions, for the different choices of switching times over a given prediction horizon. This allows selection of the best possible next switching time, which is implemented by the control agent. .The controller of the leader intersection repeats this calculations every $\Delta$ seconds (typically $\Delta=3$ to 10 seconds) in order to determine whether it should switch its traffic lights from green to orange or not, and does implement the switch if that is optimal.

The minimization of the waiting times at the leader intersections will also indicate for which intervals of switching times at the upstream intersections a good solution can be achieved at the leader intersection. Based on the optimal control selection by the leader CA this leader CA will also send as additional specification, each time it has made a decision, to each of its neighboring follower intersections, a message that sets lower and upper bounds on the switching times at the follower intersection, choosing those bounds so that the leader intersection can find a good solution, with very little wasted capacity. In practice this amounts to selecting switching times at the follower intersection so that platoons that it releases arrive at the leader at a time when it is feasible for the leader intersection to immediately give this platoon a green light, without giving red to non-empty queues, and at a time when platoons arriving from other upstream followers of the leader intersection can also use this green period. The 
leader CA thus selects not only switching times for its own traffic lights, but also messages indicating allowable intervals for switching times at each of its followers. Each follower CA will solve a local optimization problem, minimizing the average delay in its queues as averaged over all traffic flows, within the bounds imposed by the messages from its leaders. Since a follower intersection may have several leaders, the follower intersection will have to solve a constraint satisfaction problem checking if it can find switching times for its traffic lights that satisfy all its specifications. If yes it selects the best solution in terms of minimizing its local average delay. If these specifications however are contradictory and no solution can be found, then the assignment of leader agents and followers in the network will have to be modified.

\section{Applicability of leader/follower paradigm}

The proposed leader/follower paradigm is applicable under two main conditions. There must be a limited number of components that are critically loaded, and which may cause overall deterioration of the plant behavior if their load keeps increasing. Moreover these leaders must have "upstream components" that can limit the load at their downstream neighbors. In other words for each link connecting two components there must be one of the components that determines uniquely how much load flows over the link.

A number of other case studies could be used to validate the leader/follower paradigm. Consider control of freeway traffic by using on-ramp metering (limiting the flow of traffic entering a freeway at different on-ramps by traffic lights) where the components are the sections of the freeway in between on-ramps. The design of controllers for hydrological networks, whether for control of irrigation channels or for flood control, also can be approached via the leader/follower paradigm. Logistics networks and flexible manufacturing problems could also possibly be studied in this way.

\section{AVOIDING VOLTAGE COLLAPSE IN POWER SYSTEMS USING COORDINATED MODEL PREDICTIVE CONTROL}

\section{A. Problem description}

The power of the CMPC coordination control method has been investigated by applying it to secondary voltage control for an electric power transmission system. Indeed the size of the power system, sometimes called the largest man-made machine, as well as the partitioning of the network as a result of deregulation and liberalization, have led to partitioning the control of the power systems into controllers for multiple regions, each region operated by an independent TSO acting on one or several CAs. This naturally leads to a modular model of the system. The network $G$ consists of a set $V$ of nodes, each node representing a generator/load bus to which some generators and some loads are connected. A hybrid automaton $\mathrm{HA}_{\mathrm{m}}$ (including in fact also some algebraic equations abstracting the fast dynamics) models the evolution of voltage and active and reactive power in the $\mathrm{m}$-th node. Active and reactive power is transmitted between nodes via a set $E$ of tie-lines numbered $\ell=1, \ldots, \# \mathrm{E}$, and each node in $V$ is connected to these transmission lines via transformers. The transmitted power on each line depends on the port variables $V_{m \ell}(t)$, the voltage at the point where $\operatorname{Link}_{\ell}$ is connected to node $m$, and the current $I_{\ell}(t)$ flowing through the transmission line $\operatorname{Link}_{\ell}$.

The winding ratio of the transformers connecting a node and a link to each other determines the ratio of the voltage at in node $H A_{m}$ to the voltage at the port linking this node to transmission link $\operatorname{Link}_{\ell}$. Voltages can be controlled by adjusting the winding ratio of these transformers. By adjusting the voltages these adjustable transformers also adapt the current through the tie-lines, and the active and reactive power transmitted between neighboring regions.

Load tap changing transformers (LTCs) are slowly acting discrete devices operating at time scale of several seconds that allow to adjust this ratio. Typical LTCs adjust their winding ratio in small steps, over a narrow range of values (e.g. 10 steps up and down, with a maximal change of 5\% from nominal. Adjustment typically occur by taking one step up or down at some points in time. Technical limitations of LTCs impose a delay of approximately $10 \mathrm{sec}$ between a request to move the tap position up or down, and the actual change of the winding ratio. The case study discussed in this essay uses LTC adjustment as actuators for each controllable input-output $H A_{m}$, and given the inherent time delay in the LTC actions it is reasonable to adjust the winding ration approximately every $10 \mathrm{sec}$.

\section{B. $\quad$ Coordinated model predictive voltage control}

Under traditional deadband control of LTC, the LTC winding ratio is adjusted as a function of the local voltage of the connected bus, in order to locally maintain its associated bus voltage between a lower and upper bound. This totally uncoordinated operation of LTCs in a multi-area power system often leads to destablizing interactions and increases the risk of blackouts. One scenario is that a local LTC move in one area initiates, possibly undesirable, moves in LTCs located in neighboring areas and so on. If this evolution continues, there may eventually be several neighboring LTCs that may reach their maximum (or minimum) physical limits, withdrawing their voltage support from that point onwards. Interaction of LTC controls is (one of) the most likely driving mechanisms for voltage control but also a possible cause of voltage collapse in the long-term. The long-term voltage instability often occurs when, in addition to the saturation of LTCs, other discrete-event mechanisms such as over excitation limiter (OXL) of synchronous generators also become activated imposing limits on their maximum reactive power capability. Thus, it seems rather intuitive to equip each local voltage CA with an anticipation and coordination feature on what will be the future behavior of a power system, ensuring that the control actions do not lead to violation of LTC and OXL constraints in the foreseeable future.

In this way each $C A_{m}$, implemented as a $\mathrm{MPC}_{m}, m=1, \ldots$ $\# V$ \}, selects its LTC moves not only by taking local information and the local model $H A_{m}$ into account. Knowing 
the planned changes of neighboring LTCs (at the other end of transmission lines ending in bus m) $C A_{m}$ also anticipates the expected change in import/export of reactive power over the transmission lines it is connected to, knowing approximately the model of its neighbors. This approximate prediction of the effect of neighboring control actions allows each control agent to only change the LTC position when absolutely necessary. This minimizes the risk of LTC saturation.

At the same time each CA can anticipate to some extent the risk that neighboring nodes will not be able to further support the voltage control due to reaching an overexcitation limit or a limit on their LTCs. Note by the way that the messages needed for coordination in this case study are extremely simple: all that needs to be transmitted is a simple ternary sequence \{up,nochange,down\} requiring less than $2 . N$ bits for an $\mathrm{N}$-step prediction horizon.

Reference [2] illustrates, via simulation of the realistic-size well-known Nordic32 test system equipped with control agents for different regions, the performance improvement that can be achieved by using CMPC. The performance of the proposed CMCP scheme is compared with both uncoordinated decentralized MPC and uncoordinated decentralized classical deadband control of LTCs, in order to identify the distinct contribution of local anticipation and feedback coordination to improved performance. The robustness of the CMPC against measurement noises and modeling errors are shown by introducing errors in the model used by the control agents for local anticipation. It turns out that the feedback nature of the MPCs provides enough robustness against those errors. The reader is referred to [2] for a careful analysis of the behavior of the Nordic-32 system, under some severe perturbations.

\section{B. Other applications of CMPC}

The CMPC paradigm can be applied to many networked systems, provided a sufficiently detailed model of the local component is available to each $\mathrm{CA}$ in order to locally anticipate the effects of future control actions. Moreover each CA must have an approximate model of its neighbors allowing it to understand how the planned control actions of its neighbors will influence its predicted trajectories. This is in principle possible for almost every imaginable networked system, but the computational cost may be quite high since the prediction window must be long enough to include all the possible delayed effects that could lead to destabilizing loops. In an urban traffic network with a Manhattan grid structure for example one should consider the effect of a red/green switching of a traffic lights at intersection $\mathrm{W}$ on its Northern neighbor $\mathrm{N}$, which influences the Eastern neighbor $\mathrm{E}$ of $\mathrm{N}$, and in turn the Southern neighbor $\mathrm{S}$ of $\mathrm{E}$, which eventually influences again the Western neighbor W of S. Assuming as a simple example that vehicles require approximately one red/green cycle time to drive to the next intersection, this implies that the full effect of a decision to switch traffic lights at $\mathrm{S}$ is felt only after 4 cycles. But this then implies that each local CA must implement an MPC with a control horizon of length 4 cycle times, leading to a very complicated combinatorial optimization problem. This is the reason why the leader/follower paradigm was proposed for the urban traffic control case study of the preceding section, rather than the CMPC paradigm. On the other hand the CMPC paradigm can handle more complicated interactions between neighbors.

\section{FURTHER READING}

The research surveyed in this paper has been reported on partly already in some papers. For the leader/follower application to urban traffic see [1]. The CMPC approach to voltage control has been detailed in the paper [2].

The purpose of this paper is to introduce an extension to the classical control design problem, where one single loop at a time is designed, to a design methodology that can handle many more feedback loops, and that is therefore much more sensitive to destabilizing loops. While classical design tools, like Lyapunov and Nyquist, can show how to stabilize one single loop, novel approaches are needed for networks. The main issue is the trade-off between additional "coordinating" information exchange between neighboring CAs and the need to make the overall system robust both to model uncertainty and to failures of the communication network.

\section{REFERENCES}

[1] N. Marinica and R. Boel: A leader/follower approach for distributed coordination of interacting components, in Proceedings of the 20th International Symposium on Mathematical Theory of Networks and Systems, Melbourne, 2012.

[2] M. Moradzadeh, R. Boel and L. Vandevelde, "Voltage Coordination in Multi-Area Power Systems via Distributed Model Predictive Control," IEEE Trans. on Power Syst., vol. 28, no.1, pp. 513-521, Feb. 2013. 